\title{
A incorporação de calcário em sistema plantio direto consolidado reduz o estoque de carbono em macroagregados do solo
}

\author{
The incorporation of limestone in consolidated no-tillage system reduces carbon stock in soil \\ macroaggregates
}

\author{
Renato Yagi ${ }^{I}$ Jonez Fidalski" ${ }^{\text {II }}$ Cássio Antonio Tormena ${ }^{\mathrm{III}}$
}

\section{- NOTA -}

\section{RESUMO}

Estoques de carbono em macroagregados do solo possuem relação com a taxa de sequestro de C no solo em sistema plantio direto. Objetivou-se avaliar os estoques de carbono em macroagregados $(12,5-19,0 \mathrm{~mm})$ em função do revolvimento ocasional elou da calagem de um Latossolo Vermelho Distroférrico típico textura muito argilosa, sob sistema plantio direto há 17 anos em Pato Branco, sudoeste do Paraná. A partir de outubro de 2009, foi avaliado um experimento em parcelas sub-subdivididas, tendo como parcelas, épocas de amostragens após 6, 12 e 20 meses do revolvimento do solo elou calagem; nas subparcelas, manejos de solo, sem e com revolvimento do solo com uma aração e uma gradagem; e, nas sub-subparcelas, niveis de calagem, sem e com calcário para elevar o índice de saturação por bases para $70 \%$. Dos 6 aos 12 meses, a aplicação de calcário em superfície ou incorporado aumentou os estoques de $C$ dos macroagregados na camada de 0,10-0,20m. Após 20 meses, a incorporação de calcário em solo reduziu os estoques de $C$ dos macroagregados somente na camada de 0-0,10m. Assim, a calagem na superfície é fundamental para a manutenção e aumento dos estoques de $\mathrm{C}$ em macroagregados de solo sob sistema plantio direto, mantendo-o consolidado.

Palavras-chave: matéria orgânica do solo, preparo ocasional, sequestro de carbono.

\section{ABSTRACT}

Carbon stocks in soil macroaggregates are related to the rate of soil C sequestration in no-tillage system. This study aimed to evaluate carbon stocks in macroaggregates $(12.5$ to $19.0 \mathrm{~mm})$ as a function of the occasional tillage and/or liming of a Hapludox loamy clayey under no-tillage for 17 years in Pato Branco, Paraná southwest, Brazil. Starting from October 2009, it was evaluated an experiment with split-split plots, with plots as sampling dates after 6, 12 and 20 months of the occasional tillage and/or liming;

\begin{abstract}
the subplots as soil management, with and without occasional soil tillage with plowing and disking; and as sub-subplots, liming levels, with and without limestone to raise the base saturation index to $70 \%$. From 6 to 12 months, the limestone application on surface or incorporated increased $C$ stocks of macroaggregates in the 0.10-0.20m layer. After 20 months, the incorporation of limestone in soil reduced $C$ stocks in macroaggregates only in the $0-0.10 m$ layer. Thus, the surface liming is essential for the maintenance and enhancement of $C$ stocks in macroaggregates of no-tillage system, keeping it consolidated.
\end{abstract}

Key words: soilorganic matter, occasional tillage, carbon sequestration.

Antigos paradigmas técnicos voltam a ser avaliados, visando à manutenção da sustentabilidade do sistema plantio direto(SPD), a exemplo da incorporação de calcário ao solo. Por exemplo, o revolvimento de um Argissolo Vermelho Distrófico típico de textura franco argilo arenosa sob SPD, realizado a cada quatro anos com aração e gradagens para a incorporação de calcário, não afetou os teores de carbono (C) orgânico total do solo após 12 anos da intervenção mecânica (MARCOLAN \& ANGHINONI, 2006). No mesmo solo, com a terceira reaplicação de calcário após 12 anos, os teores de $\mathrm{C}$ orgânico total do solo na camada de 0-0,15m permaneceram inalterados após 12, 24, 36e 48 meses da incorporação do corretivo (MARCOLAN et al., 2007).

Ademais, também tem sido avaliado o preparo ocasional do solo em SPD, aquele que é caracterizado por uma única intervenção mecânica

\footnotetext{
IÁrea de Solos (ASO), Instituto Agronômico do Paraná (IAPAR). Rodovia do Café, km 496, s/n, CP 129, 84002-970, Ponta Grossa, PR, Brasil. E-mail: ryagi@ iapar.br. Autor para correspondência.

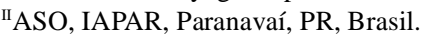

IIIDepartamento de Agronomia, Universidade Estadual de Maringá (UEM), Maringá, PR, Brasil. Recebido 06.03.14 Aprovado 21.03.14 Devolvido pelo autor 12.06.14 CR-2014.0339
} 
em área com mais de 10 anos sob SPD, sendo sugerida como prática para incrementar o potencial do solo em sequestrar C em regiões frias (QUINCKE et al., 2007). Assim, um indicador da taxa de sequestro de $\mathrm{C}$, com base em massa equivalente de solo, como os estoques de $\mathrm{C}$ em macroagregados (FERREIRA et al., 2012), poderia caracterizar o impacto positivo ou negativo de um revolvimento ocasional para incorporação de calcário ao solo em um SPD consolidado. O objetivo deste trabalho foi avaliar a influência da calagem e do revolvimento do solo nos estoques de $\mathrm{C}$ em macroagregados de um Latossolo Vermelho Distroférrico típico textura muito argilosa, sob SPD há 17 anos.

Um experimento foi implantado em 13/10/2009 na Estação Experimental do Instituto Agronômico do Paraná (IAPAR) em Pato Branco, sudoeste do Estado do Paraná (2607'16"S e $52^{\circ} 39^{\prime} 37^{\prime \prime} \mathrm{W}$; altitude de $\left.730 \mathrm{~m}\right)$. A área estava sob SPD conduzida continuamente com rotação de culturas há 17 anos, com relevo plano a suave ondulado, apresentando na camada de $0-0,20 \mathrm{~m}$ : teor de argila de $620 \mathrm{~g} \mathrm{~kg}^{-1}$, teor médio de $\mathrm{C}$ de $23 \mathrm{~g} \mathrm{dm}^{-3}$, e índice de saturação por bases de $57 \%$ e valores médios de densidade do solo nas camadas de $0-0,10$; $0,10-0,20$ e $0,20-0,30 \mathrm{~m}$, respectivamente, de 1,26 ; 1,30 e $1,20 \mathrm{Mg} \mathrm{m}^{-3}$.

O delineamento experimental foi $\mathrm{O}$ de blocos casualizados no esquema de parcelas sub-subdivididas, com quatro repetições, tendo como parcelas, épocas de amostragens de solo após implantação do experimento, após 6, 12 e 20 meses, sendo as amostragens realizadas após colheitas de milho (Zea mays L.), aveia preta (Avena strigosa Schreb.) e soja (Glycine max (L.) Merr.), respectivamente; nas subparcelas, o revolvimento ou não do solo, realizado em 13/10/2012 com arado de 4 discos, com 32" de diâmetro, seguida de gradagem niveladora com grade de 32 discos e 14" de diâmetro, na camada de $0-0,20 \mathrm{~m}$; e nas sub-subparcelas: a aplicação ou não de $1,7 \mathrm{Mg} \mathrm{ha}^{-1}$ de calcário dolomítico (PRNT $=100 \%$ ) para elevar o índice de saturação por bases a $70 \%$.

Nas camadas de $0-0,10 ; 0,10-0,20$ e 0,20 $0,30 \mathrm{~m}$ foram coletados blocos de solo com largura de $0,10 \mathrm{~m}$, comprimento de $0,20 \mathrm{~m}$ e espessura de $0,10 \mathrm{~m}$, os quais foram destorroados manualmente, obtendo-se 40 macroagregados de solo, os quais foram selecionados com peneiras metálicas de 12,5 e $19,0 \mathrm{~mm}$ de abertura. Os agregados foram secos à sombra, moídos e tamisados em peneira de $2 \mathrm{~mm}$ para a determinação dos teores de C orgânico (PAVAN et al., 1992). Amostras indeformadas de solo também foram coletadas nas camadas de $0-0,10 ; 0,10-0,20$ e $0,20-0,30 \mathrm{~m}$ para quantificar a densidade do solo (CLAESSEN, 1997).

Os estoques de $\mathrm{C}$ no solo foram calculados conforme descrito por ELLERT \& BETANY (1995), utilizando-se a camada de 0,20-0,30m de profundidade como referência (LEE et al., 2009). Os resultados de estoques de $\mathrm{C}$ em macroagregados das camadas de 0-0,10 e 0,10-0,20m foram submetidos à análise de variância (Teste F) e à comparação de médias, utilizando o teste de Tukey.

Os resultados de estoques de C apresentaram interação tripla significativa (épocas de amostragens, manejo do solo e calagem) na camada de $0-0,10 \mathrm{~m}(\mathrm{P} \leq 0,10)$; interação dupla entre as épocas de amostragens com manejo do solo $(\mathrm{P}<0,05)$ e calagem $(\mathrm{P}<0,05)$ na camada de 0,10-0,20m.

Dos 12 aos 20 meses, os estoques de C dos macroagregados na camada de $0-0,10 \mathrm{~m}$ diminuíram (20\%) somente no tratamento com incorporação de calcário ao solo; por outro lado, os estoques de $\mathrm{C}$ mantiveram-se constantes ao longo do tempo com a aplicação de calcário na superfície do solo (Figura 1a). Resultados de estoques de C do solo obtidos no presente trabalho são distintos daqueles que consideram somente os teores de $\mathrm{C}$ do solo (MARCOLAN \& ANGHINONI, 2006; MARCOLAN et al., 2007), pois alterações nestes últimos não foram observadas com a incorporação esporádica de calcário em SPD consolidado. Dessa forma, a incorporação mecânica de calcário ao solo atuou na oxidação microbiológica dos estoques de C da camada superficial do solo.

Quando não associado à calagem, o revolvimento ocasional do solo não reduziu os estoques de $\mathrm{C}$ dos macroagregados da camada de 0-0,10m, mantendo-se constante nas amostragens de solo subsequentes à intervenção mecânica (Figura 1a). Tanto no sistema de cultivo convencional quanto no SPD, a humificação da matéria orgânica do solo aumenta em profundidade (FAVORETTO et al., 2008), podendo manter os estoques de $\mathrm{C}$ em macroagregados de solo mesmo após o revolvimento, devido à maior recalcitrância das substâncias húmicas expostas na camada superficial do solo, além da incorporação destas e de materiais orgânicos, que compensam a oxidação adicional, devido o revolvimento.

Acalagem, seja ela feita na superficialmente ou incorporada ao solo, aumentou (40\%) os estoques de $C$ na camada de $0,10-0,20 \mathrm{~m}$ dos 6 aos 12 meses (Figura 1b). Mesmo aplicado à superfície, os produtos da dissociação do calcário, em particular os íons $\mathrm{Ca}^{2+}$, migraram em profundidade do solo, possivelmente 


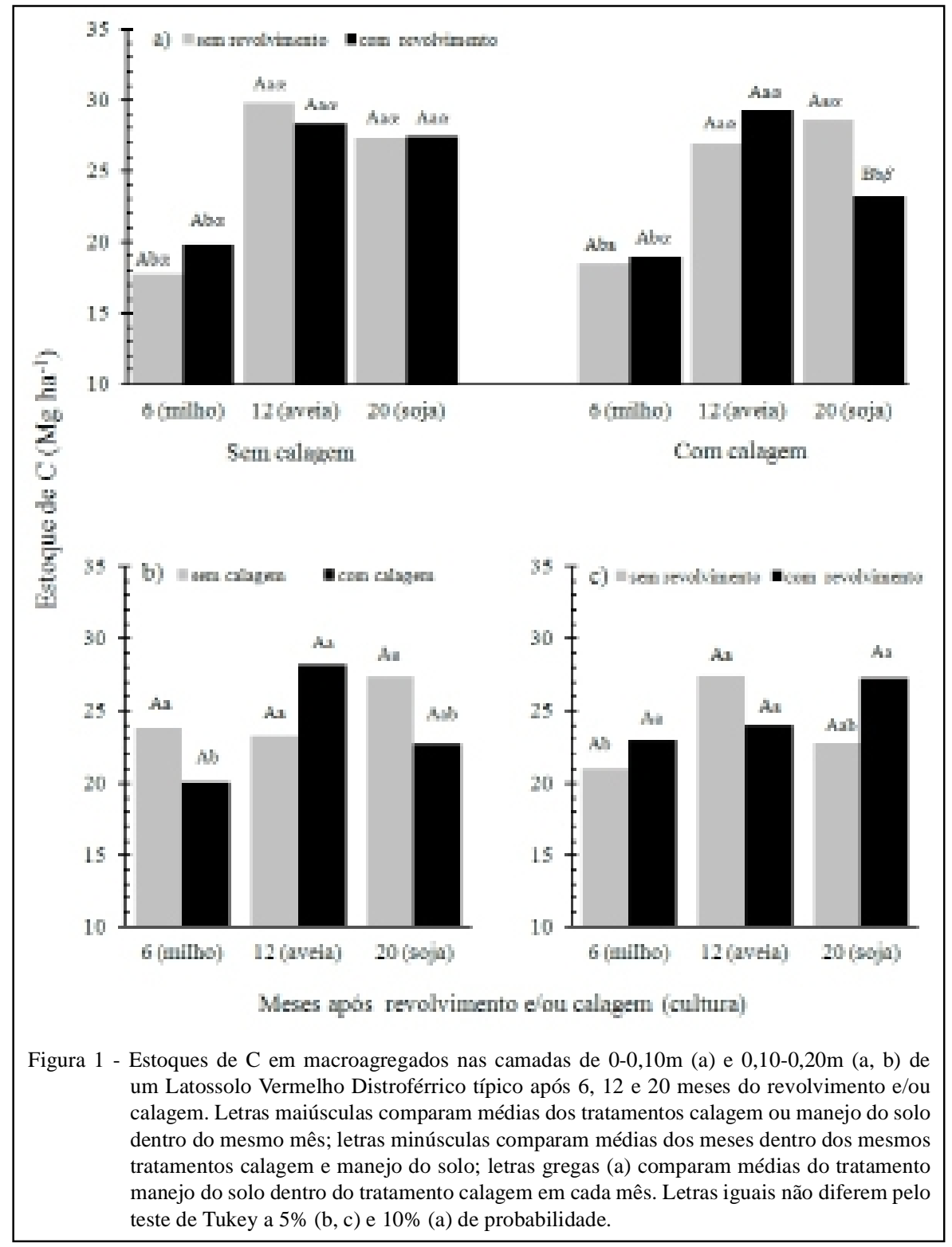

por intermédios de ácidos orgânicos de baixo peso molecular (MIYAZAWA et al., 1996) e de possíveis formações de compostos com sulfato e nitrato, bem como fisicamente associado ao movimento de água em macroporos. Dessa forma, ao se concentrar na camada de $0,10-0,20 \mathrm{~m}$, o $\mathrm{Ca}^{2+}$ pode ter atuado como ponte de cátion entre moléculas orgânicas e minerais da fração argila, aumentando os estoques de C do solo (BRIEDIS et al., 2012). Ao contrário da camada de 0-0,10m, o aumento nos estoques de $\mathrm{C}$ na camada de 0,10-0,20m com a incorporação de calcário pode ser associado às condições menos favoráveis de decomposição microbiana na sub-superfície do solo, que, associadas à incorporação dos resíduos vegetais com o revolvimento do solo com alto teor de argila, propiciaram proteção química e física de complexos organo-minerais a partir de pontes de cátions.

Houve aumento (31\%) dos estoques de $\mathrm{C}$ dos agregados da camada de 0,10-0,20m, dos 6 aos 12 meses, quando o SPD foi mantido continuamente, o que não ocorreu quando o solo foi revolvido (Figura 1c). Assim, também pode ter havido contribuição do aporte de biomassas de raízes de milho e aveia preta na camada de $0,10-0,20 \mathrm{~m}$ do solo sob SPD sem revolvimento do solo; por outro lado, em solo revolvido, uma possível homogeneização e diluição dos teores de $\mathrm{C}$ na camada de $0-0,20 \mathrm{~m}$ resultaram na ausência de aumento significativo dos estoques de $\mathrm{C}$ na camada de 0,10 $0,20 \mathrm{~m}$ entre estas duas amostragens. 


\section{AGRADECIMENTOS}

À Fundação Araucária e ao Conselho Nacional de Desenvolvimento Científico e Tecnológico (CNPq) pelas bolsas de produtividade em pesquisa concedidas ao segundo (448/2013) e terceiro autor (303624/2013-2), respectivamente.

\section{REFERÊNCIAS}

BRIEDIS, C. et. al. Soil organic matter pools and carbonprotection mechanisms in aggregate classes influenced by surface liming in a no-till system. Geoderma, v.170, p.80-88, 2012. Disponível em: <http://www.sciencedirect.com/science/article/pii/ S0016706111002965>. Acesso em: 09 mai. 2014. ?doi:10.1016/j. geoderma.2011.10.011.

CLAESSEN, M.E.E. (Org.). Manual de métodos de análise de solo. 2.ed. rev. atual. Rio de Janeiro: Embrapa-CNPS, 1997. 212p. (Embrapa-CNPS. Documentos, 1)

ELLERT, B.H.; BETTANY, J.R. Calculation of organic matter and nutrients stored in soils under contrasting management regimes. Canadian Journal of Soil Science, v.75, n.4, p.529-538, 1995. Disponível em: <http://pubs.aic.ca/doi/pdf/10.4141/cjss95-075>. Acesso em: 10 jul. 2006.

FAVORETTO, C.M. et al. Determination of humification degree of organic matter of an Oxisol and of its organo-mineral fractions. Química Nova, v.31. n.8, p.1994-1996, 2008. Disponível em: <http://www.scielo.br/scielo.php?pid=S0100$40422008000800015 \&$ script $=$ sci_arttext $>$. Acesso em 24 nov. 2012. doi: $10.1590 /$ S0100-40422008000800015.

FERREIRA, A.O. et al. Relação de estratificação como indicador do sequestro de carbono em macroagregados de Latossolo sob plantio direto. Ciência Rural, v.42, n.4, p.646-652, 2012. Disponível em: <http://www.scielo.br/scielo.php?script=sci_arttext\&pid=S0103-
$84782012000400011 \& \operatorname{lng}=$ pt\&nrm=iso\&tlng=en>. Acesso em: 09 mai. 2014. doi: 10.1590/S0103-84782012000400011.

LEE, J. et al. Determining soil carbon stock changes: Simple bulk density corrections fail. Agriculture, Ecosystems \& Environment, v.134,n.3,p.251-256, 2009. Disponível em: <http:// www.sciencedirect.com/science/article/pii/S0167880909002254>. Acesso em: 09 mai. 2014.doi: 10.1016/j.agee.2009.07.006.

MARCOLAN, A.L.; ANGHINONI, I. Atributos físicos de um Argissolo e rendimentos de culturas de acordo com o revolvimento do solo em plantio direto. Revista Brasileira de Ciência do Solo, v.23, n.1, p.163-170, 2006. Disponível em: <http://www.scielo.br/ scielo.php?script=sci_arttext $\&$ pid $=$ S0100-06832006000100016 $>$. Acesso em: 01 dez. 2012. doi: 10.1590/S0100-06832006000100016.

MARCOLAN, A.L. et al. Recuperação de atributos físicos de um Argissolo em função do seu revolvimento e do tempo de semeadura direta. Brasileira de Ciência do Solo, v.31, n.1, p.571-579, 2007. Disponível em: <http://www.scielo.br/scielo.php?script=sci_artt ext\&pid=S0100-06832007000300017> . Acesso em 2 dez. 2012. doi: 10.1590/S0100-06832007000300017.

MIYAZAWA, M. et al. Effects of addition of crop residues on the leaching of $\mathrm{Ca}$ and $\mathrm{Mg}$ in Oxisols. In: INTERNATIONAL SYMPOSIUM ON PLANT-SOIL INTERACTIONS AT LOW $\mathrm{pH}$, 1996, Belo Horizonte, MG. Abstracts... Belo Horizonte: Sociedade Brasileira de Ciência do Solo/EMBRAPA-CPAC, 1996. p.8.

PAVAN, M.A. et al. Manual de análise química do solo e controle de qualidade. Londrina: IAPAR, 1992. 40p. (Circular técnica, 76).

QUINCKE, J.A. et al. Occasional tillage of no-till systems: carbon dioxide flux and changes in total and labile soil organic carbon. Agronomy Journal, v.99, n.4, p.1158-1168, 2007. Disponível em: <https://www.agronomy.org/publications/aj/abstracts/99/4/1158>. Acesso em: 09 mai. 2014 doi: 10.2134/agronj2006.0317. 